\title{
Percepción social del aprovechamiento turístico en áreas naturales protegidas
}

\author{
Social perception of tourism use in protected natural areas \\ Percepção social do uso turístico em áreas naturais protegidas
}

Lucinda Arroyo Arcos ${ }^{1}$, María de Jesús Moo Canul' ${ }^{1}$, Romano Gino Segrado Pavón ${ }^{1}$

1Universidad de Quintana Roo, Quintana Roo, Chetumal, México

Palabras clave:

Percepción;

Turismo;

Aprovechamiento;

Cozumel;

Áreas Naturales Protegidas.

Keywords:

Perception;

Tourism;

Cozumel;

Use;

Protected Natural Areas.

Palavras-chave:

Percepção;

Turismo;

Cozumel;

Uso;

Áreas Naturais Protegidas.

Revisado por pares.

Recibido en: 13/08/2020.

Aprobado en: 19/02/2021.

Editor:

Glauber Eduardo de Oliveira Santos
Resumen

La percepción social puede constituirse en un factor influyente en la creación, promoción y ejecución de las políticas públicas de aprovechamiento de los espacios naturales con uso turístico. El objetivo general del estudio fue evaluar los factores más influyentes en la percepción social de los residentes de la isla de Cozumel acerca del aprovechamiento turístico de las Áreas Naturales Protegidas. Se aplicó un enfoque cuantitativo, con la técnica de encuesta y una muestra aleatoria representativa por conglomerados, para evaluar la percepción de impactos hacia la naturaleza, el aprovechamiento sustentable y el turismo, con un modelo de ecuaciones estructurales. Los resultados revelan una percepción negativa sobre el aprovechamiento turístico de las Áreas Naturales Protegidas, pero se reconocen los beneficios económicos y una preocupación social por la conservación. El factor más influyente en la percepción de la población sobre los impactos positivos de las Áreas Naturales Protegidas fue "Beneficios del turismo". Se concluye que los beneficios económicos son el factor predominante en la percepción social de la población de Cozumel. Se sugiere que las políticas públicas de aprovechamiento turístico deben incentivar programas educativos que expliquen la relación entre conservación y beneficios económicos.

Abstract

Social perception can be an influential factor in the creation, promotion and implementation of public policies for the use of natural spaces with tourist use. The overall objective of the study was to evaluate the most influential factors in the social perception of residents of the island of Cozumel about the tourist use of Protected Natural Areas. A quantitative approach was applied, with the survey technique and a representative random sample by conglomerates, to evaluate the perception of impacts towards nature, sustainable use and tourism, with a model of structural equations. The results reveal a negative perception on the tourist use of the Protected Natural Areas, but the economic benefits and a social concern for conservation are recognized. It is concluded that economic benefits are the predominant factor in the social perception of the population of Cozumel. It is suggested that public policies for tourism development should encourage educational programs that explain the relationship between conservation and economic benefits.

Resumo

A percepção social pode constituir-se num factor influente na criação, promoção e execução das políticas públicas de aproveitamento dos espaços naturais com uso turístico. O objetivo geral do estudo foi avaliar os fatores mais influentes na percepção social dos residentes da ilha de Cozumel sobre o aproveitamento turístico das Áreas Naturais Protegidas. Aplicou-se uma abordagem quantitativa, com a técnica de pesquisa e uma amostra aleatória representativa por conglomerados, para avaliar a percepção de impactos para a natureza, o aproveitamento sustentável e o turismo, com um modelo de equações estruturais. Os resultados revelam uma percepção negativa sobre o aproveitamento turístico das Áreas Naturais Protegidas, mas se reconhecem os benefícios econômicos e uma preocupação social pela conservação. O fator mais influente na percepção das pessoas sobre os impactos positivos das Áreas Naturais Protegidas foi "Benefícios do turismo". Conclui-se que os benefícios econômicos são o fator predominante na percepção social da população de Cozumel. Sugere-se que as políticas públicas para o desenvolvimento do turismo devem incentivar programas educacionais que expliquem a relação entre conservação e benefícios econômicos. 
Como citar: Arroyo A., L.; Moo C., M. J.; Segrado P., R. G. (2022). Percepción social del aprovechamiento turístico en áreas naturales protegidas. Revista Brasileira de Pesquisa em Turismo, São Paulo, 16, e-2222. http://dx.doi.org/10.7784/rbtur.v16.2222

\section{INTRODUCCIÓN}

La percepción social de los residentes es importante para gestionar adecuadamente los impactos turísticos en los aspectos ecológicos, sociales y económicos, para conservar y promover los atractivos turísticos y apoyar el desarrollo sustentable, se constituye como elemento significativo derivado de la interrelación con la población anfitriona (Garau-Vadell et al., 2014) y del apoyo social que cualquier actividad económica requiere, especialmente cuando existe inquietud o ideas preconcebidas respecto a las potenciales afectaciones negativas.

Como elemento constitutivo de los conflictos sociales (Lara y Ocampo, 2002), la percepción social surge de las experiencias, necesidades, expectativas, valores, motivaciones, relaciones interpersonales, estímulos y creencias, que permiten contextualizar, interpretar y comprender el entorno social (Lamb et al., 2009), así como establecer formas de interacción en respuesta a determinadas condiciones de distintos acontecimientos sociales. Para Pitcher (2016), la percepción presenta múltiples aspectos desde la base de la conciencia e implica la comprensión y definición de la realidad, que influye en las actitudes, conductas e interacción social, como resultado de la información y el proceso de la misma relacionada a la acción de cada persona.

Aunque contraproducentes en ocasiones, la contribución de la percepción social al propio grupo es el involucramiento con sugerencias de propuestas o respuestas según los estímulos recibidos (Menzel y Maner, 2010); su aporte trasciende hacia la comprensión de los procesos, la complejidad del motivo, inferencia cognitiva, y el comportamiento en los encuentros con otros, que contribuye a generar imaginarios sociales acerca de una situación, así como crear estereotipos sobre ciertos grupos de visitantes (e.g. etnia, nacionalidad o edad) los cuales influyen en las expectativas e incluso la conducta de anfitriones y visitantes.

Cuando los anfitriones reciben a los turistas y se realizan interacciones entre ambas partes, una percepción positiva de los residentes es importante para lograr un nivel elevado de satisfacción de los visitantes y consolidar la imagen turística de un destino amigable. Por otra parte, una incorrecta percepción social podría vincular al turismo con problemas internos de la comunidad receptora, cuya causalidad se atribuirá a los visitantes. Entonces, una percepción errónea o distorsionada, podría afectar al destino y su dinámica turística, así como disminuir el potencial de colaboración social que pueden otorgar las comunidades locales hacia la conservación de las áreas naturales protegidas (Alessa et al., 2003).

Según Laing (2008) el apoyo social al turismo permite aumentar los beneficios económicos, la satisfacción de los visitantes y la capacidad de carga, sin impactar negativamente al espacio natural. Como propulsor de acciones, la percepción social puede motivar la gobernanza compartida o el apoyo público para la conservación y aumentar los ingresos para las comunidades aledañas a los espacios naturales (Buckley, 2002; Moore y Weiler, 2009). La percepción social incluso puede usarse para la promoción turística, como forma de promover la conservación (Jamal y Stronza, 2009).

Las comunidades anfitrionas conocen su entorno, son susceptibles a los impactos negativos crónicos causados por los turistas en los espacios protegidos y perciben los cambios en la naturaleza (Andereck et al., 2005), particularmente en aquellos destinos donde existen niveles elevados de pobreza y se utiliza al turismo como estrategia económica, sin considerar aspectos ecológicos o sociales, lo que implica un deterioro o degradación para los espacios protegidos a largo plazo, a cambio de un beneficio económico de corto plazo.

Para cambiar esta situación causada principalmente por motivos económicos, es necesario una nueva conducta y percepción social con respecto al uso de la naturaleza (Scheffer et al., 2001), especialmente en el caso de las actividades turísticas, que pueden tener relación directa con daños a la biodiversidad y al entorno natural, como también a la calidad del paisaje o las experiencias en espacios terrestres o marinos (Godschalk, 2004; Hopwood et al., 2005), por medio del aprovechamiento sustentable, que reconoce la dependencia humana hacia los recursos 
naturales para la subsistencia, pero acepta el uso con respeto a la estructura ecológica, integridad funcional, paisajes y capacidad de carga de los ecosistemas.

La literatura científica relacionada con la percepción de los residentes sobre el turismo es amplia y con enfoques diversos: hacia la gobernanza (Almeida-García et al., 2016; Araujo Gastal y Dall'Agnol, 2012), planificación (Afthanorhan et al., 2017; Brida et al., 2013; Ernoul, 2009), beneficios (Sinclair-Maragh et al., 2015; Sita y Nor, 2015), o desde las áreas naturales protegidas (Bauer, 2003; Canto-Silva y Da Silva, 2017; Pérez-Calderón et al., 2020; Vodouhê et al., 2010). Sin embargo, los estudios dirigidos a evaluar la percepción social hacia el aprovechamiento sustentable de recursos naturales, tales como los arrecifes, han sido limitados, en los cuales destaca Pham (2012). Estudios que vinculan los arrecifes con la sustentabilidad han sido dirigidos principalmente hacia la satisfacción de los turistas (e.g. Uyarra et al., 2009), las buenas prácticas ambientales (e.g. Lucrezi y Saayman, 2017) o beneficios económicos (e.g. Vianna et al., 2012).

El análisis de la percepción social de los residentes acerca del aprovechamiento sustentable de espacios naturales protegidos con arrecifes ha sido poco analizado y existen lagunas en el conocimiento acerca de los destinos turísticos y la percepción social sobre esta actividad económica y sus impactos ecológicos y sociales. Para investigar al respecto, se eligió como caso de estudio la isla de Cozumel, destino turístico del Caribe mexicano que en 2017 recibió un total de 739,518 turistas, presenta una ocupación hotelera anual promedio de 67.8\% (SEDETUR, 2018).

Este destino especializado en el turismo marino, tiene como atractivo principal el Área Natural Protegida del Parque Nacional Arrecifes de Cozumel (PNAC), que en 2016 registró 335,985 visitantes, cifra que incrementó a 368,648 en 2017 (Hernández Hernández, 2018). En este espacio son comunes las actividades de nado, esnórquel y buceo en los arrecifes, las cuales generan beneficios económicos y sociales para la comunidad local. Sin embargo, debido a que el uso turístico puede presentar contradicciones entre la conservación y el aprovechamiento, las políticas públicas deben evaluar la percepción social sobre el tema, información que podría ser utilizada para tomar decisiones (Ramírez Cavassa, 2002).

La pregunta de investigación fue ¿cómo perciben los residentes de Cozumel el aprovechamiento turístico de los arrecifes del Parque Nacional Arrecifes de Cozumel (PNAC)? Se plantearon dos hipótesis: 1) Se establece que la población anfitriona en contacto directo con los turistas presenta una percepción positiva acerca del aprovechamiento sustentable en los arrecifes del Parque Nacional Arrecifes de Cozumel (PNAC); 2) Se establece que la protección de los arrecifes es el factor más influyente en la percepción social de la población respecto al uso turístico. El objetivo general del estudio fue evaluar los factores más influyentes en la percepción social de los residentes de la isla de Cozumel acerca del aprovechamiento turístico de las Áreas Naturales Protegidas.

\section{INTERCAMBIO SOCIAL}

La teoría del intercambio social (TIS) encuentra su fundamento en la teoría de la elección racional, que propone acciones y decisiones razonadas, fundadas en percepciones de costo-beneficio, con una comparación entre alternativas. El utilitarismo, el funcionalismo y el conductismo se encuentran fuertemente asociadas con esta teoría (Cialdini y Goldstein, 2004; Zaki et al., 2011). En cuanto a los destinos turísticos, dicha teoría propone que la actitud de la población local se ve influenciada de manera positiva o negativa por la percepción de los resultados del turismo, derivado de un intercambio entre residentes y visitantes (Andereck et al., 2005).

Gursoy y Rutherford (2004) identificaron que los impactos ecológicos, sociales y económicos son importantes e influyen en las actitudes de los pobladores hacia los turistas. Para Ap (1992) la actividad turística implica un intercambio de recursos entre las partes involucradas, con beneficios mutuos. En esta dinámica de reciprocidad los residentes brindan, además de su tiempo, un apoyo al desarrollo turístico, hospitalidad y tolerancia hacia los impactos causados por el turismo, mismos que requieren la participación y el compromiso de la comunidad.

El análisis del intercambio social se ha realizado desde la sociología y antropología (Cook et al., 2013). Los supuestos clave son los siguientes: 1) La conducta presenta una serie de intercambios; 2) Las personas esperan obtener el máximo beneficio y minimizar los costos; 3) En ciertas circunstancias, se aceptarán algunos costos a cambio de otras recompensas o estímulos; 4) Recibir un beneficio implica la retribución (Cook et al., 2013). Sin embargo, las partes podrían considerar que el intercambio no es recíproco y aun así mantener la interacción, sin que exista obligación entre las partes afectadas.

La expectativa de reciprocidad es el fundamento del intercambio social y los beneficios son interpretados como situaciones o experiencias emocionales o físicas que generan satisfacción: mientras que los costos son cualquier 
pérdida, de relaciones, de situaciones, disgustos o afectación al patrimonio tangible o intangible del individuo o grupo (Cook et al., 2013). Para Frémeaux y Michelson (2011) múltiples aspectos humanos no económicos también influyen para lograr el intercambio, mientras que para Ostrom (1998) la reciprocidad requiere coordinación para producir los resultados deseados de gobernanza.

Según Ap (1992) la percepción de la comunidad local hacia el turismo, tiene una influencia desde el punto de vista de los beneficios que se reciben de forma individual o grupal, así como del análisis mental que otorga una aprobación directa o indirecta de forma temporal, según sean las condiciones que impulsan o consolidan la relación entre las partes y tienden a favorecer el intercambio, ya que en los destinos turísticos, el intercambio social podría no es negociado entre las partes, ya que las imposiciones o exigencias por distintos motivos determinan la actividad (Wang y Chen, 2015), aunque se requiera un acuerdo concreto (Coulson et al., 2014).

Los estudios acerca de la teoría del intercambio social se han centrado en el empoderamiento local (Boley et al., 2014), la fortaleza de la teoría (Rasoolimanesh et al., 2015; Ward y Berno, 2011), las normas de hospitalidad (Coulson et al., 2014), con un enfoque predominante hacia variables socio-económicas y las preferencias locales por el turismo grupal o individual (Gursoy et al., 2010), con una discreta relación hacia los espacios naturales, que representan los atractivos turísticos principales en destinos insulares.

Los espacios naturales protegidos implican una mínima intervención humana para la conservación, sin embargo, por motivos turísticos se permiten distintos usos, con sus consecuentes impactos ecológicos, económicos y sociales, por lo que a medida que aumenta la tendencia del turismo y recreación en contacto con la naturaleza, se ejerce más presión en la conservación de las áreas naturales protegidas (Eagles et al., 2017) generando conflictos de intereses entre distintos grupos sociales por causas de conservación o recreación.

\section{METODOLOGÍA}

La zona de estudio se constituyó con la isla de Cozumel, ubicada en el estado de Quintana Roo, en la zona del Caribe. Presenta una extensión aproximada de $48 \mathrm{~km}$ de norte a sur y 14,8 km de este a oeste (Instituto Nacional Estadística y Geografía, 2010). De acuerdo con los datos de la Encuesta Intercensal del INEGI (2015), el municipio cuenta con 86415 habitantes. La Isla presenta cinco áreas naturales protegidas: Parque Natural Laguna Chankanaab, la Laguna Colombia, la Reserva Ecológica Punta Sur, el Parque Nacional Arrecifes de Cozumel y el Área de Protección de Flora y Fauna isla Cozumel.

Entre los atractivos de la isla destacan los arrecifes de coral del Parque Nacional Arrecifes de Cozumel (mapa 1), en los cuales se pueden observar las prácticas cotidianas tales como el buceo, esnórquel y recorridos de observación en embarcaciones. La Isla cuenta con 65 centros de alojamiento, con un total de 4,098 habitaciones (SEDETUR, 2018) y diversos servicios de apoyo a los visitantes.

Para comprobar las hipótesis, se realizó una investigación de tipo descriptiva con enfoque cuantitativo, para la recolección de datos se utilizó un muestreo aleatorio representativo por conglomerados, aplicando la técnica de encuesta con un cuestionario cuya unidad de estudio fueron los residentes de casas habitaciones de la isla de Cozumel. Todos los barrios del centro urbano de Cozumel fueron considerados en este estudio. Los criterios de inclusión de los informantes fueron: un año de residencia en la isla, tener 18 o más años de edad, si había más de un habitante en la casa seleccionada se otorgaba preferencia al jefe o jefa de familia.

El cuestionario se conformó por una serie de 25 ítems, según un instrumento aplicado previamente por Pham (2012), Ross (1992) y Johnson et al. (1994), adaptado para las Áreas Naturales Protegidas de Cozumel. Las respuestas presentaban una escala de Likert de cinco opciones de negativo a positivo: "totalmente en desacuerdo" (1), "en desacuerdo" (2), "ni de acuerdo ni en desacuerdo" (3), "de acuerdo" (4) y "totalmente de acuerdo" (5). Se recolectaron las características demográficas de los informantes y datos de seis dimensiones vinculadas al aprovechamiento sustentable en arrecifes turísticos: 1) Impactos turísticos positivos [tres preguntas], 2) Impactos turísticos negativos [cuatro preguntas], 3) Evaluación de impactos turísticos [dos preguntas], 4) Aprovechamiento sustentable [seis preguntas], 5) Protección de los arrecifes [seis preguntas], 6) Beneficios del turismo [cuatro preguntas]. 
Mapa 1 - Sitios de buceo del Parque Nacional Arrecifes de Cozumel

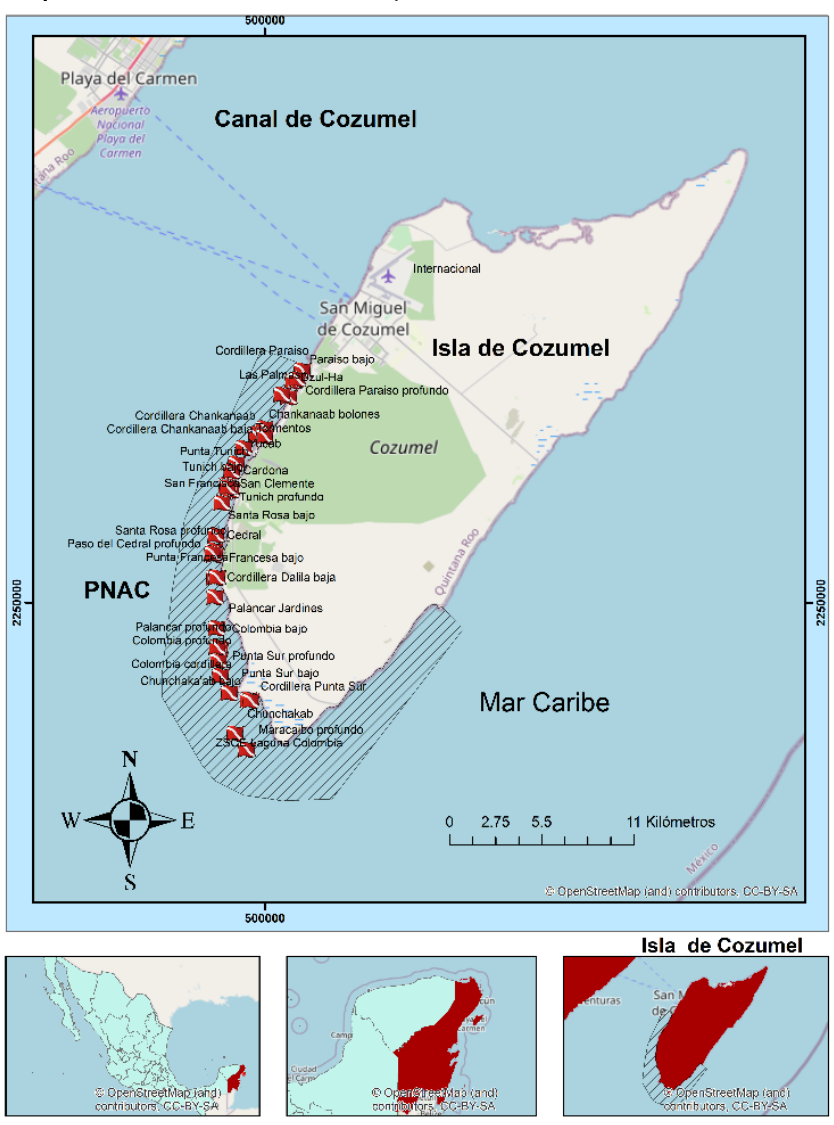

Fuente: datos propios, con el software OpenStreetMap, 2021.

En 2015 se aplicaron 501 cuestionarios, de los cuales fueron válidos 449. El muestreo presenta un nivel de confianza del $95 \%$ y $3 \%$ de margen de error. Para el procesamiento de los datos se utilizó el software estadístico SPSS, mediante el cual se hizo el análisis descriptivo y de la varianza con el ANOVA de un factor para la comparación de las medias estadísticas de la población de estudio, para contrastar la hipótesis nula de que las medias de los grupos de población son iguales y cuando el resultado es estadísticamente significativo es que hay alguna diferencia entre los grupos (Lai y Kelley, 2012).

Para el análisis inferencial se diseñó un modelo estructural que incorpora las dimensiones analizadas y fue procesado mediante el software Smart PLS. El modelo fue de ecuaciones estructurales (SEM) mediante mínimos cuadrados parciales, previa comprobación de la fiabilidad y validez. Este tipo de modelo se utiliza para predecir y explorar porque es "especialmente útil cuando el objetivo de la investigación es probar teorías o avanzar en el desarrollo de las mismas” (Máynez Guaderrama y Vargas Salgado, 2019).

Para comprobar la fiabilidad del modelo se revisaron las cargas factoriales y el Alfa de Cronbach, utilizando como límite de validación los valores mayores a 0.7 (Nunnally y Bernstein, 1994). Además, se revisó que el índice de fiabilidad compuesta (Oliden y Zumbo, 2008) fuera superior a 0.7. Posterior a la revisión del modelo propuesto, fue necesario eliminar los indicadores que no cumplieron estos criterios, conservando únicamente los indicadores de las dimensiones cuyas cargas fueron significativamente distintas de cero y cercanas o superiores a 0.6 (Bagozzi y Yi, 1988) ya que se buscó mantener la validez nomológica y que cumplieran los criterios mínimos de fiabilidad.

Para el análisis de validez discriminante, se verificó que la varianza extraída promedio (AVE) estuviera por encima del punto de corte 0.5 (Fornell y Larcker, 1981). También se revisó que la varianza extraída promedio de cada constructo fuera superior al cuadrado de las correlaciones entre pares de los otros constructos (Fornell y Larcker, 1981), así como la revisión de las cargas cruzadas fuese acorde al constructo teórico correspondiente (Götz et al., 2010). En cuanto a la no colinealidad, se validó mediante el factor de inflación de la varianza (FIV), cuyos valores internos y externos fueron menores al punto de corte de 10 (Diamantopoulos et al., 2008). 


\section{RESULTADOS}

Las edades de los encuestados oscilaron entre 18 a 55 años, y un porcentaje menor (7\%) lo conformaron personas mayores a 55 años. En relación con el tiempo que llevan residiendo en la isla de Cozumel, la mayoría (82\%) tiene más de 18 años y el 18\% tiene entre 2 y 15 años viviendo en la isla. De acuerdo con el Anuario Estadístico de Quintana Roo (2017), el 66\% de la población económicamente activa está inserta en el sector servicios y un 20\% labora en el comercio, por lo tanto, existe una relación directa o indirecta con ésta actividad.

Como primera parte del análisis de los resultados descriptivos se agrupó cada uno de los ítems del cuestionario de acuerdo con las dimensiones del modelo teórico. En la tabla 1 se observa que la media de la evaluación sobre su percepción acerca de los impactos positivos hacia la naturaleza fue neutral, ya que las evaluaciones medias estuvieron cercanas a la calificación del rubro tres, que en la escala utilizada del 1 al 5 , indica que no estaban de acuerdo ni en desacuerdo, con relación a la percepción de los impactos positivos que otorga el Área Natural Protegida sobre la naturaleza.

Tabla 1 - Dimensión: Percepción de los impactos positivos en la naturaleza

\begin{tabular}{|c|c|c|c|c|c|}
\hline & Ítems $(n=449)$ & Mínimo & Máximo & Media & Desv. típ. \\
\hline A & El turismo contribuye a la protección de la naturaleza en las ANP. & 1 & 5 & 3.02 & 1.007 \\
\hline B & El turismo ha mejorado la naturaleza de la isla en muchas formas. & 1 & 5 & 2.97 & 1.005 \\
\hline C & El turismo ha mejorado la apariencia visual de la isla. & 1 & 5 & 3.36 & .993 \\
\hline
\end{tabular}

La tabla 2 presenta la opinión sobre la dimensión de aprovechamiento sustentable y cuyos resultados muestran en primer lugar con una media de 2.88 "el valor primario de los arrecifes es proveer beneficios a los humanos" y en segundo lugar con una media de 2.71 "las personas deben usar los arrecifes exclusivamente para beneficio humano", de forma general con las afirmaciones planteadas se observa una percepción negativa con una media de 2.66 .

Tabla 2 - Dimensión: Aprovechamiento sustentable

\begin{tabular}{|c|c|c|c|c|c|}
\hline & Ítems $(n=449)$ & Mínimo & Máximo & Media & Desv. típ. \\
\hline$\overline{\mathrm{D}}$ & Las personas deben usar los arrecifes exclusivamente para beneficio humano. & 1 & 5 & 2.71 & 1.069 \\
\hline $\mathrm{E}$ & Las necesidades humanas son más importantes que los arrecifes. & 1 & 5 & 2.68 & .952 \\
\hline $\mathrm{F}$ & El valor primario de los arrecifes es proveer beneficios a los humanos. & 1 & 5 & 2.88 & 1.024 \\
\hline G & $\begin{array}{l}\text { El uso turístico de los arrecifes es más importante que proteger las especies que } \\
\text { habitan allí. }\end{array}$ & 1 & 5 & 2.34 & .927 \\
\hline $\mathrm{H}$ & Los arrecifes existen para ser usados por las personas & 1 & 5 & 2.73 & 1.005 \\
\hline
\end{tabular}

En relación con la opinión sobre la protección de los arrecifes, en la tabla 3 se observa que las respuestas se ubican cerca del número 4 (opción "Muy de acuerdo"), esto implica que los encuestados están de acuerdo con las afirmaciones planteadas enfocadas a valorar, cuidar y proteger a los arrecifes, entre las que destaca el cuidarlos para las generaciones futuras con una media de 4.35 .

Tabla 3 - Dimensión: Protección de los arrecifes

\begin{tabular}{|c|c|c|c|c|c|}
\hline & Ítems ( $n=449)$ & Mínimo & Máximo & Media & Desv. típ. \\
\hline $\mathrm{I}$ & $\begin{array}{l}\text { Los arrecifes deben ser protegidos por su propio valor y no sólo para satisfacer a } \\
\text { las personas o los turistas. }\end{array}$ & 1 & 5 & 4.14 & .630 \\
\hline J & Los arrecifes deben tener derechos similares a los derechos de las personas. & 1 & 5 & 3.63 & .845 \\
\hline $\mathrm{K}$ & El uso turístico de los arrecifes no debe permitirse si se les causan daños serios. & 1 & 5 & 3.91 & .762 \\
\hline $\mathrm{L}$ & Es importante cuidar los arrecifes para las generaciones futuras. & 2 & 5 & 4.35 & .613 \\
\hline M & Los arrecifes son importantes con o sin uso turístico. & 2 & 5 & 4.08 & .660 \\
\hline
\end{tabular}

La tabla 4, presenta la opinión sobre variables de la dimensión de beneficios del turismo; éstas se encontraron en 3.65 en la escala que del 1 al 5, lo cual implica que los encuestados estuvieron de acuerdo con las afirmaciones planteadas, entre las cuales destaca con una media de 3.96 aquella que afirma que el gobierno debería controlar el turismo en los arrecifes para mejorar los beneficios y reducir los perjuicios. 
Tabla 4 - Dimensión: Beneficios del turismo

\begin{tabular}{|c|c|c|c|c|c|}
\hline & Ítems & Mínimo & Máximo & Media & Desv. típ. \\
\hline$\overline{\mathrm{N}}$ & Si vienen más turistas al arrecife sería mucho mejor para todos. & 1 & 5 & 3.31 & 1.074 \\
\hline 0 & $\begin{array}{l}\text { El gobierno debería hacer más esfuerzos para mejorar la infraestructura para los tu- } \\
\text { ristas. }\end{array}$ & 1 & 5 & 3.62 & .991 \\
\hline $\mathrm{P}$ & $\begin{array}{l}\text { El gobierno debería controlar el turismo en los arrecifes para mejorar los beneficios } \\
\text { y reducir los perjuicios. }\end{array}$ & 1 & 5 & 3.96 & .746 \\
\hline Q & La comunidad debería apoyar el desarrollo turístico del arrecife. & 1 & 5 & 3.71 & .866 \\
\hline
\end{tabular}

Para identificar si el trabajo turístico o el contacto con el turismo incidían en la opinión de los encuestados sobre este aspecto, se realizó un ANOVA de un factor. Los resultados se observan en la tabla 5. De acuerdo con las diferencias de medias, la afirmación sobre "si el turismo ha mejorado la naturaleza de la isla en muchas formas", presenta diferencia estatisticamente significativa (0.031), esto implica que la opinión de la comunidad cambia dependiendo del grado de contacto con los turistas.

Tabla 5 - ANOVA Percepción de impactos y contacto con el turista

\begin{tabular}{|c|c|c|c|c|c|c|}
\hline$(n=449)$ & & Suma de cuadrados & gl & Media cuadrática & $F$ & Sig. \\
\hline \multirow{3}{*}{$\begin{array}{l}\text { El turismo contribuye a la protección de la naturaleza } \\
\text { en las ANP. }\end{array}$} & Inter-grupos & .198 & 1 & .198 & .195 & .659 \\
\hline & Intra-grupos & 454.533 & 447 & 1.017 & & \\
\hline & Total & 454.731 & 448 & & & \\
\hline \multirow{3}{*}{$\begin{array}{l}\text { El turismo ha mejorado la naturaleza de la isla en mu- } \\
\text { chas formas. }\end{array}$} & Inter-grupos & 4.679 & 1 & 4.679 & 4.669 & .031 \\
\hline & Intra-grupos & 447.944 & 447 & 1.002 & & \\
\hline & Total & 452.624 & 448 & & & \\
\hline \multirow[t]{3}{*}{ El turismo ha mejorado la apariencia visual de la isla. } & Inter-grupos & .000 & 1 & .000 & .000 & .994 \\
\hline & Intra-grupos & 441.550 & 447 & .988 & & \\
\hline & Total & 441.550 & 448 & & & \\
\hline
\end{tabular}

En la tabla 6 se observa que, si en su trabajo el encuestado tiene contacto con los turistas, su opinión sobre si "el turismo ha mejorado la naturaleza de la isla en muchas formas", tiende a ser más negativa (2.82), que por el contrario si su trabajo no tiene contacto con el turista (3.04). Sobre las demás variables, no existe diferencia significativa en las opiniones.

Tabla 6 - ANOVA Percepción de impactos positivos y contacto con el turista

\begin{tabular}{|c|c|c|c|c|c|c|c|c|c|}
\hline \multirow[t]{2}{*}{$(n=449)$} & & \multirow[b]{2}{*}{$\mathrm{n}$} & \multirow[b]{2}{*}{ Media } & \multirow[b]{2}{*}{ Desviación típica } & \multirow[b]{2}{*}{ Error típico } & \multicolumn{2}{|c|}{$\begin{array}{l}\text { Intervalo de confianza para la } \\
\text { media al } 95 \%\end{array}$} & \multirow[b]{2}{*}{ Mínimo } & \multirow[b]{2}{*}{ Máximo } \\
\hline & & & & & & Límite inferior & Límite superior & & \\
\hline \multirow{3}{*}{$\begin{array}{l}\text { El turismo contribuye a la pro- } \\
\text { tección de la naturaleza en las } \\
\text { Áreas Naturales Protegidas. }\end{array}$} & Sí & 136 & 2.99 & 1.022 & .088 & 2.82 & 3.17 & 1 & 5 \\
\hline & No & 313 & 3.04 & 1.002 & .057 & 2.93 & 3.15 & 1 & 5 \\
\hline & Total & 449 & 3.02 & 1.007 & .048 & 2.93 & 3.12 & 1 & 5 \\
\hline \multirow{3}{*}{$\begin{array}{l}\text { El turismo ha mejorado la natu- } \\
\text { raleza de la isla en muchas for- } \\
\text { mas. }\end{array}$} & Sí & 136 & 2.82 & .983 & .084 & 2.65 & 2.98 & 1 & 5 \\
\hline & No & 313 & 3.04 & 1.009 & .057 & 2.93 & 3.15 & 1 & 5 \\
\hline & Total & 449 & 2.97 & 1.005 & .047 & 2.88 & 3.06 & 1 & 5 \\
\hline \multirow{3}{*}{$\begin{array}{l}\text { El turismo ha mejorar la apari- } \\
\text { encia visual de la isla. }\end{array}$} & Sí & 136 & 3.36 & 1.001 & .086 & 3.19 & 3.53 & 1 & 5 \\
\hline & No & 313 & 3.36 & .991 & .056 & 3.25 & 3.47 & 1 & 5 \\
\hline & Total & 449 & 3.36 & .993 & .047 & 3.27 & 3.45 & 1 & 5 \\
\hline
\end{tabular}

En la tabla 7 se realiza el análisis del ANOVA sobre las opiniones de aquellos que trabajan en turismo y de quienes no lo hacen, respecto a su percepción de impactos positivos en la naturaleza. En ninguna variable se observa significancia estadística $(p<0.05)$, es decir, no hay diferencia de opiniones entre los encuestados, trabajen o no con el turismo. 
Tabla 7 - ANOVA Percepción de impactos positivos y trabajo en turismo

\begin{tabular}{|c|c|c|c|c|c|c|}
\hline$(n=449)$ & & Suma de cuadrados & gl & Media cuadrática & $\mathrm{F}$ & Sig. \\
\hline \multirow{3}{*}{$\begin{array}{l}\text { El turismo contribuye a la protección de la natu- } \\
\text { raleza en las ANP. }\end{array}$} & Inter-grupos & 4.308 & 2 & 2.154 & 2.133 & 0.120 \\
\hline & Intra-grupos & 450.422 & 446 & 1.010 & & \\
\hline & Total & 454.731 & 448 & & & \\
\hline \multirow{3}{*}{$\begin{array}{l}\text { El turismo ha mejorado la naturaleza de la isla } \\
\text { en muchas formas. }\end{array}$} & Inter-grupos & .667 & 2 & 0.334 & 0.329 & 0.720 \\
\hline & Intra-grupos & 451.956 & 446 & 1.013 & & \\
\hline & Total & 452.624 & 448 & & & \\
\hline \multirow{3}{*}{$\begin{array}{l}\text { El turismo ha mejorado la apariencia visual de la } \\
\text { isla. }\end{array}$} & Inter-grupos & 1.948 & 2 & 0.974 & 0.988 & 0.373 \\
\hline & Intra-grupos & 439.602 & 446 & 0.986 & & \\
\hline & Total & 441.550 & 448 & & & \\
\hline
\end{tabular}

En la Figura 1 se presenta el modelo estructural depurado, con atención a todos los estándares presentados en el apartado del Método. Este modelo presenta la influencia que tiene el interés por la protección de los arrecifes, el aprovechamiento turístico y los beneficios de la actividad turística, hacia la percepción social de la población sobre los impactos positivos de las Áreas Naturales Protegidas.

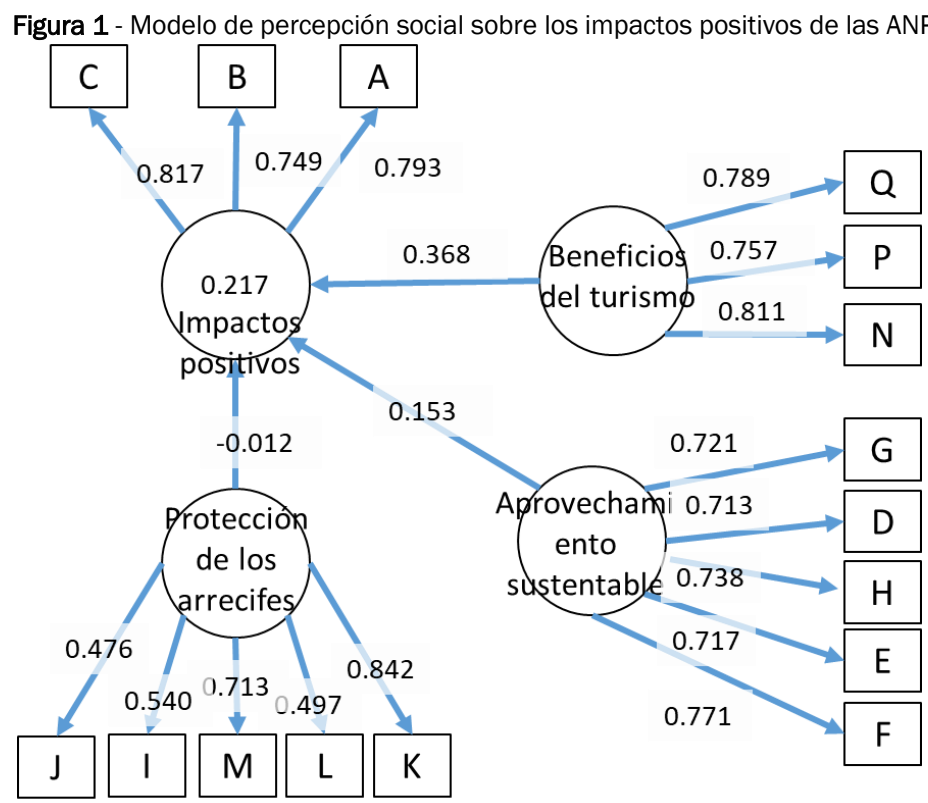

Nota: $\mathrm{n}=449$.

En relación con el grado explicativo del modelo estructural, Malhotra (2008) y Hair (2019) establecen que, obtener un $\mathrm{R}^{2}$ sobre el 0.33 es lo adecuado; en este caso se obtuvo 0.217 , que implica un grado explicativo aceptable, aunque dentro del rango débil (0.19 a 0.32). En el apartado de Discusión se evaluará esta situación.

Finalmente, se analizó la significancia estadística de las relaciones hipotéticas del modelo depurado, se presenta en la figura 2, por medio de un bootstrapping (remuestreo teórico) de 500 opciones usando el software estadístico Smart PLS.

Posterior al procesamiento y depuración del modelo probado, se analizaron las relaciones hipotéticas del modelo estructural, en donde se observó que los datos recolectados considerados en el modelo estructural (aprovechamiento sustentable y beneficios del turismo) influyen significativa y positivamente en la percepción de la comunidad sobre los impactos positivos en las ANP, situación no observada en la dimensión sobre la protección de los arrecifes. En la figura 2 se observa el análisis del modelo estructural, en donde el factor relacionado con "Beneficios del turismo" ( $\beta=7.316$; $p$ <.001) fue el que influyó con mayor peso en la percepción de la población sobre los impactos positivos de las ANP. 
Figura 2 - Factores de percepción de la población e impactos positivos de las ANP

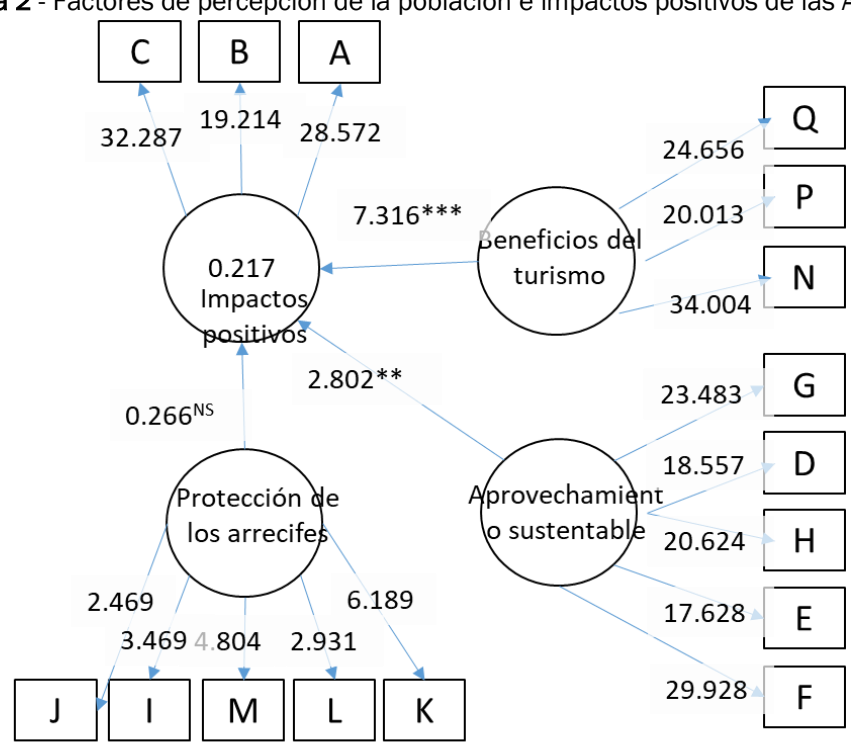

Nota: ${ }^{\text {NSNo }}$ significativo, significativo al $* * 99 \%$ y $* * * 99.9 \%$ de confianza con valores $t$ para una prueba de dos colas.

Nota: $n=449$.

De forma simplificada, la figura 3 presenta el resumen de interrelación entre los distintos componentes teóricos del modelo, en donde se puede apreciar que "Beneficios del turismo" y el "Aprovechamiento sustentable" son significativos, mientras que la protección de los arrecifes no logra significación estadística.

Figura 3 - Modelo sintético de percepción e impactos positivos de las ANP

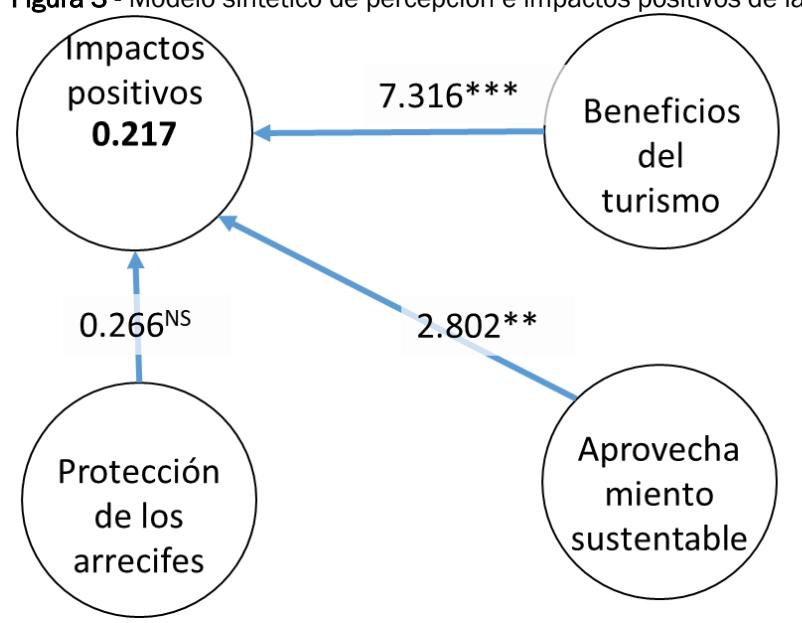

Nota: ${ }^{\text {NS}}$ No significativo, significativo al $* * 99 \%$ y ***99.9\%

de confianza con valores $t$ para una prueba de dos colas.

\section{DISCUSIÓN}

Con base en 449 cuestionarios aplicados a una muestra aleatoria por conglomerados en la isla de Cozumel y un análisis estadístico inferencial ANOVA, se establece que existe una diferencia significativa en la percepción entre las respuestas de los residentes que tienen contacto con turistas mediante su trabajo y los residentes que no, en relación al turismo y sus impactos hacia la naturaleza de la isla ( $F 4.669$ y sig. 0.031). En contraste, la percepción entre residentes que son empleados turísticos y los que no lo son, no mostró diferencia significativa en la percepción de impactos a la naturaleza. 
Estos resultados sugieren que la población de Cozumel se encuentra influida de manera positiva o negativa, según el tipo de trabajo que desempeña: mientras su contacto con el turismo sea más directo, la percepción resulta más negativa, lo cual se relaciona con los hallazgos de Andereck et al. (2005) y Cook et al. (2013). Asimismo, los resultados se vinculan con las aportaciones de Ap (1992), quien menciona que la percepción de la comunidad local hacia el turismo conlleva una aprobación o rechazo, conforme los beneficios que se reciben de manera individual o grupal (Cook et al., 2013).

A través de los ítems más relevantes de cada dimensión se puede inferir la percepción que tienen los residentes del aprovechamiento turístico de los espacios naturales. En primera instancia, dentro de la dimensión "aprovechamiento sustentable" aparece como ítem más relevante "el uso turístico de los arrecifes es más importante que proteger las especies que habitan alli", lo cual posiciona el proteger los ecosistemas arrecifales como prioridad respecto a su uso turístico. Lo anterior se complementa con el ítem "los arrecifes deben ser protegidos por su propio valor y no sólo para satisfacer las personas o los turistas", perteneciente a la dimensión "protección del arrecife", debido que los residentes apoyan su protección por los procesos naturales que se llevan a cabo intrínsecamente y no solo por su belleza paisajística y que ésta atraiga a los turistas. Por último, la dimensión "beneficios del turismo" muestra que el ítem más significativo es "el gobierno debería controlar el turismo en los arrecifes para mejorar los beneficios y reducir los perjuicios" donde se percibe el apoyo a medidas que coadyuven a las estrategias de conservación de los arrecifes, tales como normas y sanciones para avanzar hacia una buena gobernanza como lo menciona Ostrom (1998).

Estos resultados reflejan cierta inclinación por parte de los residentes hacia la protección de los recursos naturales, debido a la conciencia de los habitantes con relación a la valía y relevancia del medio natural, así como a lo propuesto por Johnson et al., (1994), quienes mencionan que a largo plazo los residentes son más conscientes del valor de sus recursos naturales para uso turístico; aunado a esto, la importancia de las percepciones radica en el uso estratégico que estas ofrecen a la implementación de políticas y planes de desarrollo de un lugar turístico (Ap, 1992). De acuerdo con Sita \& Nor (2015), en cuando a estudios de percepción enfocados a lugares turísticos, se deben tomar en cuenta las investigaciones longitudinales con el fin de observar y evaluar el ciclo de vida del destino, por lo que los presentes resultados deben complementarse con otros estudios previos o posteriores.

Los hallazgos de este estudio deben ser evaluados con atención a ciertas limitaciones: 1) las dos dimensiones ya consolidadas en otros estudios se han mostrado más robustas, significativas y relacionadas entre sí ("Beneficios del turismo" e "Impacto naturales"), mientras que las dimensiones de Aprovechamiento sustentable y Protección de arrecifes podrían incorporarse dentro de la dimensión de impactos naturales (en futuras investigaciones), más que una dimensión separada, aunque el modelo requiere ser probado con otros estudios de casos; situación que está relacionada con la carencia de estudios previos dirigidos al aprovechamiento sustentable y la conservación de arrecifes; 2) el desconocimiento de la población local acerca de las áreas naturales protegidas que existen en la Isla; 3) como estudio de caso, el alcance es regional. Como fortalezas, el tamaño muestral es grande, representativo de la comunidad local y la estandarización del instrumento permite la replicabilidad en otros destinos turísticos del arrecife Mesoamericano.

En relación al análisis inferencial resultado del modelo estructural, se presentó la discusión sobre la percepción de la población sobre los impactos positivos del turismo en las Áreas Naturales Protegidas. En este se obtuvo un R² reducido probablemente debido a que la condición de espacio natural protegido implica un acceso libre a todos los visitantes, mientras que la población local percibe que tiene un cierto derecho de exclusividad sobre estos espacios naturales, por lo que el turismo se percibe como un beneficio, pero la declaración de Área Natural Protegida ha implicado más competencia, más requisitos administrativos y de infraestructura para brindar servicios turísticos.

Por último, a través de análisis de ecuaciones estructurales, y usando el método de mínimos cuadrados parciales, se resalta el factor "beneficios del turismo" por su influencia económica significativa en la percepción de la población sobre los impactos positivos de las Áreas Naturales Protegidas. Mediante los ítems usados en este análisis los residentes perciben que a través del manejo adecuado de los ecosistemas arrecifales junto con una gestión local se podría beneficiar a más residentes por las visitas al arrecife.

\section{CONCLUSIONES}

Esta investigación propuso dos hipótesis: 1) Se establece que la población anfitriona en contacto directo con los turistas presenta una percepción positiva acerca de los impactos hacia los espacios naturales; en los resultados se rechaza esta hipótesis, puesto que ocurre lo contrario: en la medida que existe una interacción con el turista, la 
percepción tiende a ser más negativa. 2) Se establece que la protección de los arrecifes son el factor más influyente en la percepción social de la población respecto al uso turístico; en los resultados también se rechaza, debido a que "Beneficios del turismo" fue el factor con mayor significancia.

El objetivo general del estudio fue evaluar los factores más influyentes en la percepción social de los residentes de la isla de Cozumel acerca del aprovechamiento turístico de las áreas naturales protegidas. Se determinó que los factores "aprovechamiento sustentable" y "beneficios del turismo" ejercen una influencia positiva en la percepción, siendo el "beneficios del turismo" el factor con más influencia significativa. "Protección al arrecife" tuvo una influencia negativa pero una relación hipotética no estadísticamente significativa, es decir, a través del análisis inferencial se observó una percepción negativa hacía el manejo de los arrecifes, no obstante, las medias descriptivas presentaron una percepción positiva hacia la protección de los mismos.

Con base en la teoría del intercambio social se deduce que los residentes no tienen una percepción positiva hacia el aprovechamiento turístico de áreas protegidas en Cozumel en ciertos factores específicos, lo que podría derivar en actitudes, creencias, expectativas o conductas negativas que perjudiquen la experiencia de los turistas, por lo cual es necesario diseñar y aplicar medidas necesarias para minimizar la insatisfacción entre la recreación, la conservación y aprovechamiento turístico de los espacios protegidos, para aumentar el apoyo y participación de los residentes en el crecimiento turístico de Cozumel.

Las áreas naturales protegidas son esenciales dentro de la oferta turística de la Isla, por lo cual la percepción de los residentes es fundamental para gestionar adecuadamente los impactos negativos en estos espacios protegidos. Debido al incremento de servicios y actividades de ocio al interior y la zona de influencia de las Áreas Naturales Protegidas, así como limitado control y seguimiento sobre el uso turístico y recreativo, se recomienda diseñar y aplicar estrategias o políticas públicas dirigidas a la educación ambiental para las Áreas Naturales Protegidas en el destino, explicando la relación entre conservación y atractivos turísticos.

\section{AGRADECIMIENTOS}

A la Comisión Nacional de Áreas Naturales Protegidas (CONANP) por el apoyo recibido por medio del convenio de colaboración PROMANP 2014.

\section{REFERENCIAS}

Afthanorhan, A., Awang, Z. y Fazella, S. (2017). Perception of Tourism Impact and Support Tourism Development in Terengganu, Malaysia. Social Sciences, 6(3), 106. https://doi.org/10.3390/socsci6030106

Alessa, L., Bennett, S. M. y Kliskey, A. D. (2003). Effects of knowledge, personal attribution and perception of ecosystem health on depreciative behaviors in the intertidal zone of Pacific Rim National Park and Reserve. Journal of Envi-ronmental Management, 68(2), 207-218. https://doi.org/10.1016/S0301-4797(03)00068-9

Almeida-García, F., Peláez-Fernández, M. Á., Balbuena-Vázquez, A. y Cortés-Macias, R. (2016). Residents' perceptions of tourism development in Benalmádena (Spain). Tourism Management, 54, 259-274. https://doi.org/10.1016/j.tourman.2015.11.007

Andereck, K. L., Valentine, K. M., Knopf, R. C. y Vogt, C. A. (2005). Residents' perceptions of community tourism impacts. Annals of Tourism Research, 32(4), 1056-1076. https://doi.org/10.1016/j.annals.2005.03.001

Ap, J. (1992). Residents' perceptions on tourism impacts. Annals of Tourism Research, 19(4), 665-690. https://doi.org/10.1016/0160-7383(92)90060-3

Araujo Gastal, S. d. y Dall'Agnol, S. (2012). Turismo em Laguna (SC): Impactos e atitude. Revista Brasileira De Pesqui-sa Em Turismo, 6(1), 16-31. https://www.rbtur.org.br/rbtur/article/view/481

Bagozzi, R. P. y Yi, Y. (1988). On the evaluation of structural equation models. Journal of the Academy of Marketing Science, 16(1), 74-94. https://doi.org/10.1007/BF02723327

Bauer, H. (2003). Local perceptions of Waza National Park, northern Cameroon. Environmental Conservation, 30(2), 175-181. https://doi.org/10.1017/S037689290300016X

Boley, B. B., McGehee, N. G., Perdue, R. R. y Long, P. (2014). Empowerment and resident attitudes toward tourism: Strengthening the theoretical foundation through a Weberian lens. Annals of Tourism Research, 49, 3350. https://doi.org/10.1016/i.annals.2014.08.005 
Brida, J. G., Disegna, M. y Osti, L. (2013). The effect of authenticity on visitors' expenditure at cultural events. Current Issues in Tourism, 16(3), 266-285. https://doi.org/10.1080/13683500.2012.674105

Buckley, R. (2002). Surf Tourism and Sustainable Development in Indo-Pacific Islands. II. Recreational Capacity Man-agement and Case Study. Journal of Sustainable Tourism, 10(5), 425-442. https://doi.org/10.1080/09669580208667177

Canto-Silva, C. R. y Da Silva, J. S. (2017). Panorama da visitação e da condução de visitantes em Parques brasileiros. Revista Brasileira De Pesquisa Em Turismo, 11(2), 365-386. https://doi.org/10.7784/rbtur.v11i2.1286

Cialdini, R. B. y Goldstein, N. J. (2004). Social influence: Compliance and conformity. Annual Review of Psychology, 55, 591-621. https://doi.org/10.1146/annurev.psych.55.090902.142015

Cook, K. S., Cheshire, C., Rice, E. R. y Nakagawa, S. (2013). Social Exchange Theory. En J. D. DeLamater y A. Ward (Eds.), Handbooks of sociology and social research. Handbook of social psychology (pp. 61-88). Springer.

Coulson, A. B., MacLaren, A. C., McKenzie, S. y O'Gorman, K. D. (2014). Hospitality codes and Social Exchange Theory: The Pashtunwali and tourism in Afghanistan. Tourism Management, 45, 134-141. https://doi.org/10.1016/j.tourman.2014.03.019

Diamantopoulos, A., Riefler, P. y Roth, K. P. (2008). Advancing formative measurement models. Journal of Business Research, 61(12), 1203-1218. https://doi.org/10.1016/j.jbusres.2008.01.009

Eagles, P. F. J., Snyman, S. y Spenceley, A. (2017). Guidelines for tourism partnerships and concessions for protected areas: generating sustainable revenues for conservation and development: Report to the Secretariat of the Conven-tion on Biological Diversity and IUCN. IUCN. https://portals.iucn.org/library/node/46956

Ernoul, L. (2009). Residents' perception of tourist development and the environment: a study from Morocco. International Journal of Sustainable Development \& World Ecology, 16(4), 228-233. https://doi.org/10.1080/13504500902993180

Fornell, C. y Larcker, D. F. (1981). Evaluating Structural Equation Models with Unobservable Variables and Measurement Error. Journal of Marketing Research, 18(1), 39-50. https://doi.org/10.2307/3151312

Frémeaux, S. y Michelson, G. (2011). 'No Strings Attached': Welcoming the Existential Gift in Business. Journal of Business Ethics, 99(1), 63-75. https://doi.org/10.1007/s10551-011-0749-5

Garau-Vadell, J. B., Díaz-Armas, R. y Gutierrez-Taño, D. (2014). Residents' Perceptions of Tourism Impacts on Island Destinations: A Comparative Analysis. International Journal of Tourism Research, 16(6), 578-585. https://doi.org/10.1002/jtr.1951

Godschalk, D. R. (2004). Land Use Planning Challenges: Coping with Conflicts in Visions of Sustainable Development and Livable Communities. Journal of the American Planning Association, 70(1), 5-13. https://doi.org/10.1080/01944360408976334

Götz, O., Liehr-Gobbers, K. y Krafft, M. (2010). Evaluation of Structural Equation Models Using the Partial Least Squares (PLS) Approach. En V. Esposito Vinzi (Ed.), Springer handbooks of computational statistics. Handbook of partial least squares: Concepts, methods and applications (pp. 691-711). Springer. https://doi.org/10.1007/978-3-540-32827-8 30

Gursoy, D., Chi, C. G. y Dyer, P. (2010). Locals' Attitudes toward Mass and Alternative Tourism: The Case of Sunshine Coast, Australia. Journal of Travel Research, 49(3), 381-394. https://doi.org/10.1177/0047287509346853

Gursoy, D. y Rutherford, D. G. (2004). Host attitudes toward tourism. Annals of Tourism Research, 31(3), 495-516. https://doi.org/10.1016/j.annals.2003.08.008

Hair, J. F. (2019). Multivariate data analysis (Eighth edition). Cengage.

Hernández Hernández, B. (2018, 17 de agosto). Entrevista de Romano Gino Segrado Pavón [Papel]. Cozumel.

Hopwood, B., Mellor, M. y O'Brien, G. (2005). Sustainable development: mapping different approaches. Sustainable Development, 13(1), 38-52. https://doi.org/10.1002/sd.244

Instituto Nacional de Estadística y Geografía. (2017). Anuario estadístico y geográfico de Quintana Roo 2017 . INEGI. https://www.inegi.org.mx/contenido/productos/prod_serv/contenidos/espanol/bvinegi/productos/nueva estruc/anuarios 2017/702825095130.pdf

Instituto Nacional Estadística y Geografía. (2010). Censo de Población y Vivienda (2010): Perfil sociodemográfico: Estados Unidos Mexicanos: Censo de Población y Vivienda 2010. INEGl. https://www.inegi.org.mx/contenido/productos/prod serv/contenidos/espanol/bvinegi/productos/censos/poblacion/2010/perfil socio/uem/702825047610 1.pdf 
Instituto Nacional Estadística y Geografía. (2015). Número de habitantes. Quintana Roo. INEGI. http://cuentame.inegi.org. $\mathrm{mx} / \mathrm{monografias/informacion/qroo/poblacion/}$

Jamal, T. y Stronza, A. (2009). Collaboration theory and tourism practice in protected areas: stakeholders, structuring and sustainability. Journal of Sustainable Tourism, 17(2), 169-189. https://doi.org/10.1080/09669580802495741

Johnson, J. D., Snepenger, D. J. y Akis, S. (1994). Residents' perceptions of tourism development. Annals of Tourism Research, 21(3), 629-642. https://doi.org/10.1016/0160-7383(94)90124-4

Lai, K. y Kelley, K. (2012). Accuracy in parameter estimation for ANCOVA and ANOVA contrasts: Sample size planning via narrow confidence intervals. The British Journal of Mathematical and Statistical Psychology, 65(2), 350-370. https://doi.org/10.1111/j.2044-8317.2011.02029.x

Laing, J. (2008). Understanding partnerships for protected area tourism: Learning from the literature. Cooperative Research Centre for Sustainable Tourism. https://sustain.pata.org/wp-content/uploads/2014/12/90055-Laing-Partners-PA-tourismWEB.pdf

Lamb, C. W., Hair, J. F. y McDaniel, C. D. (2009). Essentials of marketing (6th ed.). South-Western; Cengage Learning.

Lara, L. y Ocampo, L. (2002). Psicología social y comunitaria: Cognición social. Usta.

Lucrezi, S. y Saayman, M. (2017). Sustainable scuba diving tourism and resource use: Perspectives and experiences of operators in Mozambique and Italy. Journal of Cleaner Production, 168, 632-644. https://doi.org/10.1016/j.jclepro.2017.09.041

Malhotra, N. K., Dávila Martínez, J. F. J. y Treviño Rosales, M. E. (2008). Investigación de mercados (5a. edición). Pearson Educación.

Máynez Guaderrama, A. y Vargas Salgado, M. M. (2019). Modelos de ecuaciones estructurales mediante mínimos cuadrados parciales: un ejemplo de su aplicación en el campo de las ciencias administrativas. En A. Máynez y V. López (Eds.), La práctica de la investigación en las ciencias administrativas (pp. 114-135). Pearson Educación. http://cathi.uacj.mx/handle/20.500.11961/8566?show=full

Menzel, A. J. y Maner, J. K. (2010). Interpersonal Perception. En I. B. Weiner y W. E. Craighead (Eds.), The Corsini en-cyclopedia of psychology (4a ed.). John Wiley. https://doi.org/10.1002/9780470479216.corpsy0461

Moore, S. A. y Weiler, B. (2009). Tourism-protected area partnerships: stoking the fires of innovation. Journal of Sus-tainable Tourism, 17(2), 129-132. https://doi.org/10.1080/09669580802582506

Nunnally, J. C. y Bernstein, I. H. (1994). Psychometric theory (3rd ed.). McGraw-Hill series in psychology. McGrawHill.

Oliden, P. E. y Zumbo, B. D. (2008). Coeficientes de fiabilidad para escalas de respuesta categórica ordenada. Psicothema, 20(4), 896-901. http://www.psicothema.com/psicothema.asp?id=3572

Ostrom, E. (1998). A Behavioral Approach to the Rational Choice Theory of Collective Action: Presidential Address, American Political Science Association, 1997. American Political Science Review, 92(1), 1-22. https://doi.org/10.2307/2585925

Pérez-Calderón, E., Prieto-Ballester, J. M., Miguel-Barrado, V. y Milanés-Montero, P. (2020). Perception of Sustainabil-ity of Spanish National Parks: Public Use, Tourism and Rural Development. Sustainability, 12(4), 1333. https://doi.org/10.3390/su12041333

Pham, L. (2012). Perceptions of Tourism Impact and Tourism Development Among Residents of Cuc Phuong National Park, Ninh Binh, Vietnam. Journal of Ritsumeikan Social Sciences and Humanities, 3, $75-92$. https://papers.ssrn.com/sol3/papers.cfm?abstract_id=2094784

Pitcher, G. (2016). Theory of Perception. Princeton University Press.

Ramírez Cavassa, C. (2002). Calidad total en las empresas turísticas. Serie Trillas turismo. Trillas.

Rasoolimanesh, S. M., Jaafar, M., Kock, N. y Ramayah, T. (2015). A revised framework of social exchange theory to investigate the factors influencing residents' perceptions. Tourism Management Perspectives, 16(6), 335345. https://doi.org/10.1016/j.tmp.2015.10.001

Ross, G. F. (1992). Resident Perceptions of the Impact of Tourism on an Australian City. Journal of Travel Research, 30(3), 13-17. https://doi.org/10.1177/004728759203000302

Scheffer, M., Carpenter, S., Foley, J. A., Folke, C. y Walker, B. (2001). Catastrophic shifts in ecosystems. Nature, 413(6856), 591-596. https://doi.org/10.1038/35098000 
SEDETUR. (2018). Indicadores turísticos de Quintana Roo 2017. SEDETUR. https://groo.gob.mx/sedetur/indicadores-turisticos

Sinclair-Maragh, G., Gursoy, D. y Vieregge, M. (2015). Residents' perceptions toward tourism development: A factorcluster approach. Journal of Destination Marketing \& Management, 4(1), 36-45. https://doi.org/10.1016/j.jdmm.2014.10.001

Sita, S. E. D. y Nor, N. A. M. (2015). Degree of Contact and Local Perceptions of Tourism Impacts: A Case Study of Homestay Programme in Sarawak. Procedia - Social and Behavioral Sciences, 211(1), 903-910. https://doi.org/10.1016/i.sbspro.2015.11.119

Uyarra, M. C., Watkinson, A. R. y Côté, I. M. (2009). Managing dive tourism for the sustainable use of coral reefs: Vali-dating diver perceptions of attractive site features. Environmental Management, 43(1), 1-16. https://doi.org/10.1007/s00267-008-9198-z

Vianna, G.M.S., Meekan, M. G., Pannell, D. J., Marsh, S. P. y Meeuwig, J. J. (2012). Socio-economic value and community benefits from shark-diving tourism in Palau: A sustainable use of reef shark populations. Biological Conservation, 145(1), 267-277. https://doi.org/10.1016/i.biocon.2011.11.022

Vodouhê, F. G., Coulibaly, O., Adégbidi, A. y Sinsin, B. (2010). Community perception of biodiversity conservation with-in protected areas in Benin. Forest Policy and Economics, 12(7), 505-512. https://doi.org/10.1016/i.forpol.2010.06.008

Wang, S. y Chen, J. S. (2015). The influence of place identity on perceived tourism impacts. Annals of Tourism Research, 52(4), 16-28. https://doi.org/10.1016/i.annals.2015.02.016

Ward, C. y Berno, T. (2011). Beyond social exchange theory. Annals of Tourism Research, 38(4), 1556-1569. https://doi.org/10.1016/i.annals.2011.02.005

Zaki, J., Schirmer, J. y Mitchell, J. P. (2011). Social influence modulates the neural computation of value. Psychological Science, 22(7), 894-900. https://doi.org/10.1177/0956797611411057.

\section{Informacións de los autores}

\section{Lucinda Arroyo Arcos}

Es profesora investigadora de carrera, adscrita a la Universidad de Quintana Roo, Cozumel. Doctora en Geografía por la Universidad Nacional Autónoma de México (UNAM), Miembro del Sistema Nacional de Investigadores (SNI 1).

Contribuciones al artículo: diseño de la investigación, revisión de la literatura, recogida de datos, discusión de los resultados.

E-mail: larroyo@uaroo.edu.mx

ORCID: https://orcid.org/0000-0001-6696-7124

\section{María de Jesús Moo Canul}

Es maestra en comunicación corporativa por la Universidad Anáhuac de Cancún. Actualmente es profesora-investigadora del Departamento de Estudios Sociales y Empresariales, División de Desarrollo Sustentable en la Universidad de Quintana Roo, Unidad Cozumel. Sus líneas de investigación actuales son estrategias de comunicación para el aprovechamiento turístico sustentable y estudios sobre satisfacción turística.

Contribuciones al artículo: concepción de la investigación, metodología, análisis y discusión de los datos.

E-mail: mariajmc@uqroo.edu.mx

ORCID: https://orcid.org/0000-0003-1849-163X

\section{Romano Gino Segrado Pavón}

Profesor investigador de la carrera de la Universidad de Quintana Roo, División de Desarrollo Sustentable. Doctorado en Ciencias Ambientales, con líneas de investigación en el área del aprovechamiento turístico sustentable en espacios naturales y límites sociales para el uso de la naturaleza.

Contribución al artículo: concepto y diseño de la investigación, revisión de la literatura científica, recolección de datos, análisis de datos, discusión, conclusión, referencias.

E-mail: romanogino@hotmail.com

ORCID: https://orcid.org/0000-0002-9923-4944 